\title{
Crecimiento de Istiophorus platypterus (Pisces: Istiophoridae) en la boca del Golfo de California
}

\author{
Reyna Alvarado-Castillo' y Roberto Félix-Uraga \\ Centro Interdisciplinario de Ciencias Marinas, Departamento de Pesquerías y Biología Marina. Apdo. postal 592 y 476. La Paz \\ B.C.S. México. C.P. 23000.
}

\author{
Recibido 12-VI-1994. Corregido 15-X-1997. Aceptado 27-X-1997
}

\begin{abstract}
Specimens (733) of Istiohporus platypterus caught by sport fishing in the southern of the California Gulf were measured. The fourth spine of the dorsal fin was collected to estimate the age and individuals from 1 to 8 years were found. The majority of the specimens were between 180 and $210 \mathrm{~cm}$ in lower jaw-fork length. The Bertalanffy growth ecuation estimated using length was: $L_{1}=203.6\left[1-e^{-(1)(t+1)(x) \cdot 5]}\right]$. Growth was shown to very fast in the early years.
\end{abstract}

Key words: Sailfish, growth, California Gulf, age, Istiophorus plaryptents.

El pez vela Istiophorus platypterus se distribuye en el Océano Pacífico oriental desde las costas de Ecuador hasta la península de Baja California, México (Kume y Joseph 1969). En México, es uno de los recursos mas atractivos de la pesca deportiva, sin embargo se han realizado pocas investigaciones sobre su biología. Se ha reportado la presencia de juveniles desde del Golfo de Tehuantepec hasta Cabo San Lucas, B.C.S. (Laurs y Nishimoto 1970, Guzmán-Arrollo y López-Ramos 1986, Aguilar-Palomino et al. 1994). También se realizaron experimentos de marcado y recaptura en el Golfo de California (Squire 1974, 1979). En un análisis de distribución de tallas de la flota deportiva que opera en Manzanillo, Colima, se encontraron organismos entre $114-208 \mathrm{~cm}$ de longitud posorbital, con una moda en $165-175 \mathrm{~cm}$ (Macías-Zamora 1993). Se reportó que el pez vela es un desovador parcial y su periodo de reproducción en el sur del Golfo de California, es durante el verano y otoño, la talla promedio a la que el $50 \%$ de las hembras son maduras es de $175 \mathrm{~cm}$ longitud ojo-furca (Hernández-Herrera 1994) y las edades encontradas fueron de 1 a 8 años con tallas entre
$155-235 \mathrm{~cm}$ de longitud furcal (Alvarado-Castillo y Félix-Uraga 1996).

En las investigaciones realizadas en el Pacífico Mexicano sobre pez vela, ninguna aborda el tema del crecimiento individual, el cual junto con las estimaciones de edad, son factores importantes para la evaluación y manejo del recurso.

Durante el periodo de 1989 a 1991, se llevaron a cabo muestreos de pez vela capturados por la flotadeportiva que opera en Manzanillo, Colima; Puerto Vallarta. Jalisco; Mazatlán, Sinaloa; Cabo San Lucas y La Paz, B.C.S. México. Cada ejemplar, se midió de la mandíbula inferior a la furca (longitud mandibular LM), y se recolectóla 4a. espina de la aleta lorsal de 733 organismos para estimar laedad mediante el conteo de bandas de crecimiento opaco-hialinas en cortes hechos en la base de las espinas (Alvarado-Castillo y Félix-Uraga 1996). Los datos deedadse agruparon por intervalo de talla de $5 \mathrm{~cm}$ y se estimó la talla promedio por edad. 
El crecimiento individual del pez vela, se describió con el modelo de von Bertalanffy, los parámetros de la ecuación de crecimiento $\mathrm{L}_{.}, \mathrm{k}, \mathrm{t}_{\mathrm{o}}$ se calcularon utilizando el método de LópezVeiga (1979), en el cual se usan las siguientes ecuaciones :

$$
\begin{gathered}
L_{h}=\frac{\left(L_{t t}\right)^{2}-L_{0} L_{t 2} K}{2 L_{t 1}-L_{t 2}-L_{0}}=\frac{\ln \left(\frac{L_{h}-L_{0}}{L_{h}-L_{t 1}}\right)}{t_{1}} \\
t_{0}=\frac{\ln \left(\frac{L_{h}-L_{0}}{L_{h}}\right)}{K}
\end{gathered}
$$

\section{CUADRO}

Frecuencia de Istiophorus platypterus por grupo de tallas y edades para el periodo 1989-1991
El valor de $\mathrm{L}_{0}$ (talla de la larva al momento de la eclosión) utilizado, fue de $2.5 \mathrm{~mm}$, que es la larva mas pequeña encontrada por Leis et al. (1987).

En la distribución de frecuencia por talla y edad se observaron organismos de 155 a $230 \mathrm{~cm}$ de LM y hasta ocho años de edad, aunque en el intervalo de 180 a $210 \mathrm{~cm} \mathrm{LM}$, también se encontraron individuos de todas las edades presentes (Cuadro 1).

Edad (años)

Longitud mandibular $(\mathrm{cm})$
$151-155$
$156-160$
$161-165$
$166-170$
$171-175$
$176-180$
$181-185$
$186-190$
$191-195$
$196-200$
$201-205$
$206-210$
$211-215$
$216-220$
$221-225$
$226-230$

Talla promedio

1
1
0
1
0
1
0
0
0
3
1
0
1
0
0
0
0

184.3

2
0
1
0
1
4
5
11
8
13
16
11
10
2
0
0
1

193.6

$\begin{array}{cc}3 & 4 \\ 1 & 0 \\ 0 & 0 \\ 1 & 1 \\ 3 & 4 \\ 9 & 6 \\ 8 & 8 \\ 17 & 21 \\ 27 & 28 \\ 29 & 40 \\ 34 & 35 \\ 29 & 50 \\ 19 & 36 \\ 15 & 15 \\ 3 & 5 \\ 1 & 4 \\ 2 & 0 \\ & \\ 195 & \end{array}$

$195.4 * \quad 197.1$

$\begin{array}{cccc}5 & 6 & 7 & 8 \\ 0 & 0 & 0 & 0 \\ 0 & 0 & 0 & 0 \\ 0 & 1 & 0 & 0 \\ 2 & 1 & 0 & 0 \\ 3 & 0 & 0 & 0 \\ 9 & 1 & 0 & 0 \\ 5 & 1 & 0 & 0 \\ 9 & 5 & 0 & 0 \\ 15 & 5 & 2 & 0 \\ 12 & 4 & 6 & 1 \\ 30 & 3 & 2 & 1 \\ 14 & 11 & 2 & 0 \\ 15 & 7 & 1 & 2 \\ 6 & 4 & 1 & 0 \\ 1 & 4 & 1 & 0 \\ 1 & 3 & 0 & 0 \\ 199.1 & 204.5 * & 203.3 & 206.8\end{array}$

199.1

*Valores utilizados para estimar los parámetros de crecimiento de la ecuación de Bertalanffy. 
De acuerdoa las estimaciones de los parámetros del modelo de Bertalanffy, $\mathrm{L}_{\mathbf{S}}=203.6 \mathrm{k}=0.8 \mathrm{t}_{0}=-$ 0.0015 (Fig. 1), se puede observar que el pez vela tiene un rápido crecimientodurante el primer año de vida, alcanzando $110 \mathrm{cmde} L M$ que representa mas del $50 \%$ de la longitud infinita. Este comportamiento ha sido reportado para esta especie en otras zonas (De Sylva 1957, Jolley 1977,Hedgepety Jolley 1983,Farber 1981 citado en Hedgepet y Jolley 1983). El crecimiento tan acelerado, puede ser una estrategia para evitar ser depredados y con el rápido incremento en talla, pueden incorporarse al stock migrante.

Se considera que la talla promedio observada para las tres primeras edades está sobrestimada (Fig. 1), debido a que los organismos aún noestán completamente reclutados a la pesca deportiva, y solo los mas grandes de estas edades son capturados. Los organismos de cuatro años en adelante, ya se han reclutado completamente, es porello, que elajustede laecuación de crecimiento es muy semejante a los valores de las tallas promedio observadas.

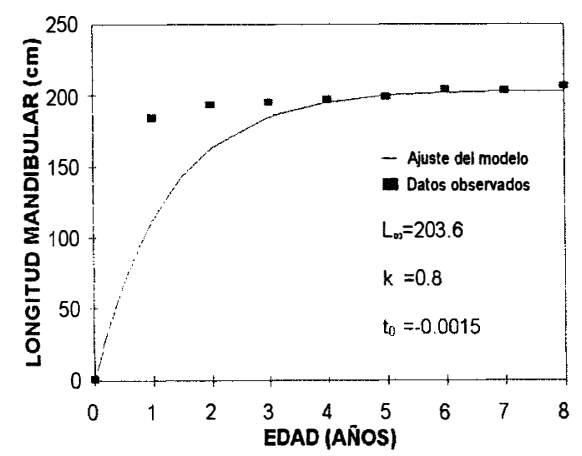

Fig 1. Curva de crecimiento del pez vela en la boca del Golfo de California, para el periodo 1989-1991.

Agradecemos a la COFAA y BDA, por el apoyo otorgado a Roberto Felix Uraga.

\section{REFERENCIAS}

Aguilar-Palomino, B, L. A. Abitia-Cardenas, J. De La CruzAgüero \& J. Rodríguez-Romero. 1994. Young sailfish Istiophorus platypterus (Osteichthyes: Istiophoridae) in Cabo San Lucas, Baja California Sur, México. Rev. Biol. Trop. 42: 391-392.

Alvarado-Castillo, R. \& R. Félix-Uraga. 1996. Determinación de laedad deIstiophorusplatypterus (Pisces: Istiophoridae) al sur del Golfode California, México. Rev. Biol Trop. 44: 233-239.

De Sylva, D.P. 1957. Studies on the age and growth of the Atlantic sailfish, Istiophorus americanus (Cuvier), using length frecuency curves. Bull. Mar. Sci. Gulf Caribb. 7: 120.

Guzmán-Arroyo,M.\& J.O.López-Ramos. 1986. Descripción y registro de juveniles de pez vela(Istiophorusplatypterus, Shaw and Nodder, 1971) (Pisces: Istiophoridae), en las costas del estado de Michoacán, Pacífico sur de México. Cien. Mar. (México) 12: 53-67.

Hedgepet, M.Y. \& J.W. Jolley. 1983. Age and growth in sailfish Istiophorus platypterus, using cross secction from the fourth dorsal fine spine, p. 131-136. In: E.D. Prince \& L.M.Pulos(eds.). Proceedingof the international workshop on age determination of oceanic pelagic fishes: tunas, billfishes, and sharks, U.S. Dep. Commer., NOAA Tech. Rep. NMFS- 8 .

Hernández-Herrera, A. 1994. Patrón reproductivo de Pez Vela (Istiophorus platypterus; Shaw y Nodder, 1791) al surdel Golfo de California. Tesis de maestría. CICIMAR, IPN, La Paz, B.C.S. México. 53 p.

Jolley, J.W. 1977. The biology and fishery of Atlantic sailfish, Istiophorusplatypterus, from Southeast Florida. Fla. Mar. Res. Publ. 28. 31 p.

Kume, S. \& J. Joseph. 1969. Size composition and sexual maturity of billfish caughtby the Japanese longline fishery in the Pacific Ocean East of $130 \infty$ W. Bull. Far. Seas. Fish Res. Lab. (Shimizu) 2: 115-162.

Laurs, M.R. \& R.N. Nishimoto. 1970. Five juvenile sailfish, Istiophorus platypterus, from the eastern tropical Pacific. Copeia 3: 590-594.

Leis, J.M., B. Goldman \& S. Ueyanagi. 1987. Distribution and abundance of billfish larvae (Pisces: Istiophoridae) in the Great Barrier Reef Lagoon and Coral Sea near Lizard Island, Australia. Fish Bull. 85: 757-765. 
López-Veiga, E.C. 1979. Fitting Von Bertalanffy growth curves in Short-lived fish species. A new approach. Invest. Pesq.; 42: 179-186.

Macias-Zamora, R. 1993. Relaciones entre la pesca deportiva y comercial de pez vela (Istiophorus platypterus) en el Pacíficomexicano, Tesis de maestria. CICIMAR, IPN, La Paz, B.C.S. México. 71 p.

Squire, J.L. Jr. 1974. Migration patterns of Istiophoridae en the Pacific Ocean as determined by cooperative tagging programs for six species of billfishes on the Central
Pacific Ocean. Part 2, p 226-237. In: R.S. Shomura and F. Williams (eds.), Proceeding of the international billfish symposium, Kailua-Kona,. U.S. Dep Commer., NOAA Tech. Rep. NMFS SSRF-675.

Squire, J.L. Jr. 1979. Billfish newsletter. Southwest Fisheries Center. Cooperative marine game fish tagging program \& Pacific billfish angler survey. National Oceanic \& Atmospheric Administration. NMFS. 17 p. 